\title{
Analysis of second outbreak of COVID-19 after relaxation of control measures in India
}

\author{
Xinchen Yu • Guoyuan Qi $\mathbb{D} \cdot$ Jianbing Hu
}

Received: 24 June 2020/Accepted: 28 September 2020/Published online: 10 October 2020

(C) Springer Nature B.V. 2020

\begin{abstract}
At present, more and more countries have entered the parallel stage of fighting the epidemic and restoring the economy after reaching the inflection point. Due to economic pressure, the government of India had to implement a policy of relaxing control during the rising period of the epidemic. This paper proposes a compartment model to study the development of COVID-19 in India after relaxing control. The Sigmoid function reflecting the cumulative effect is used to characterize the model-based diagnosis rate, cure rate and mortality rate. Considering the influence of the lockdown on the model parameters, the data are fitted using the method of least squares before and after the lockdown. According to numerical simulation and model analysis, the impact of India's relaxation of control before and after the inflection point is studied. Research shows that adopting a relaxation policy prematurely will have disastrous consequences. Even if the degree of relaxation is only $5 \%$ before the inflection point, it will increase the number of deaths by $15.03 \%$. If the control is relaxed after the inflection point, the higher
\end{abstract}

X. Yu · G. Qi ( $($ )

Tianjin Key Laboratory of Advanced Technology of Electrical Engineering and Energy, Tiangong University, Tianjin 300387, China

e-mail: guoyuanqisa@qq.com

J. $\mathrm{Hu}$

School of Mechanical Engineering, Tiangong University, Tianjin 300387, China degree of relaxation, the more likely a secondary outbreak will occur, which will extend the duration of the pandemic, leading to more deaths and put more pressure on the health care system. It is found that after the implementation of the relaxation policy, medical quarantine capability and public cooperation are two vital indicators. The results show that if the supply of kits and detection speed can be increased after the control is relaxed, the secondary outbreak can be effectively avoided. Meanwhile, the increase in public cooperation can significantly reduce the spread of the virus, suppress the second outbreak of the pandemic and reduce the death toll. It is of reference significance to the government's policy formulation.

Keywords COVID-19 · Relaxing control · Compartment model $\cdot$ Inflection point .

Second outbreak

\section{Introduction}

More than 200 countries and regions worldwide have been suffering from COVID-19 since the outbreak, and the confirmed cases are still increasing worldwide. The international community is working hard to adopt global cooperation and commitments to solve this complex problem, so as to better prepare for the pandemic [1]. As of June 22, 2020, the total 
number of confirmed cases reached 8,860,331 all over the world, including 465,740 deaths [2].

According to research, the new coronavirus is highly contagious and has human-to-human characteristics [3]. The infected person will first enter the incubation period. Patients in the incubation period will not have any symptoms and are also infectious [4]. Through clinical trials of inpatients, it was found that the patients showed symptoms consistent with viral pneumonia during the onset of COVID-19, the most common being fever, cough, sore throat and fatigue [5]. Therefore, early detection of symptomatic patients for nucleic acid detection and isolation, contact tracing and large-scale social isolation can greatly reduce the spread of the virus [6]. In China, due to timely and strict control measures, the epidemic has been effectively controlled. Earlier, Europe once thought it was the main epicenter of the epidemic. Since various European countries began to take measures to lockdown cities in March, the current epidemic has been controlled to some extent. In May, most European countries have entered the parallel stage of fighting the epidemic and restoring the economy, just like Italy, France and Spain. At present, the numbers of daily diagnosed cases in the USA and Brazil are still high and have become the countries with the most severe epidemic.

The first confirmed diagnosis of COVID-19 was in Kerala, India, on January 30, 2020. As of now (June $22,2020)$, the country has reported a total of 440,450 confirmed cases, 248,137 recovery and 14,015 deaths [7]. To slow down the spread of COVID-19, the Indian government began to implement a national lockdown on March 25. The Ministry of the Interior announced on May 30 that the ongoing national lockdown measures would continue to be extended for another month to June 30. Meanwhile, it announced a reopening timetable indicating that from June 8, non-strictly controlled areas would allow religious venues, hotels and restaurants to be opened on the premise of maintaining social distance [8]. According to the data reported by the WHO daily report, the Indian COVID-19 epidemic is still on the rise. Still, due to economic pressure, the government has to relax some of its control. This paper analyzes the impact of COVID-19 in India after the relaxation of control through the established compartmental model and how to prevent the possible second outbreak.
Statistical and mathematical models have been used to study the spread of viruses because of the dynamic characteristics of accuracy and reliability. In addition, when modeling methods cannot describe the actual behavior of the system itself, complexity science and information systems can greatly help save lives [9]. Regarding the COVID-19 pandemic, based on statistical research, Perc et al. [10] proposed a simple iterative method to predict the number of COVID-19 cases, which provided a preliminary reference and guiding principles for preventing the deterioration of the pandemic.

Many researchers also gave analysis and prediction of the development trend of the pandemic based on the SEIR (Susceptible, Exposed, Infectious, Recovered) model and SEIR-like models [11-17]. Fang et al. used the parameterized SEIR model to simulate the spread dynamics of the COVID-19 outbreak and the impact of different control measures. The model fitted the data before February 29 in China, and the trend curve of the effective reproductive number was drawn [11]. Wu et al. considered the total traffic flow from Wuhan to major cities in China and other countries in the SEIR model, estimated the scale of the epidemic in Wuhan based on the number of cases exported from Wuhan to cities outside mainland China, and forecasted the extent of the domestic and global public health risks of epidemics [12]. Anastassopoulou et al. [13] used the discrete SIRD (Susceptible, Infectious, Recovered, Dead) model to estimate the basic reproduction number and the model-based fatality and recovery ratio. Zhao et al. established the SUQC (Susceptible, Un-quarantined Infected, Quarantined Infected, Contained Infected) model to characterize the dynamics of COVID-19, and used parametric control measures to illustrate the impact of prevention and control efforts on the epidemic [14]. Rong et al. [15] developed a new SEIR-type compartment model to study the effect of delay in diagnosis on disease transmission. Mandal et al. developed the SEQIR model in [16], introduced isolation categories and government interventions to mitigate disease transmission, and also formulated an optimal control problem and determined the optimal control measures. Huang et al. [17] adopted a comprehensive SEIR model to simulate the clinical data of COVID-19 in Spain and investigated the risk of the easing of the control measure. 
In the above literature and recent COVID-19 research, it is noted that most of the parameters in the mathematical model are constant. The parameters that characterize the diagnosis rate, cure rate and mortality rate in the model should change as the medical level improves and should reflect the cumulative effect for the cumulative variables. Besides, many studies only use data on confirmed cases and death cases in parameter fitting, which is incomplete for the study of pandemic development. Since lots of countries have adopted blockade measures during the prevention and control of epidemics, sudden changes in parameters caused by the blockade of cities should be considered. How to adjust the parameters corresponding to the sudden change is problematic. Currently, India is in the growth stage of the epidemic, and research on the impact of relaxation of control on the transmission dynamics of COVID19 in India is very limited. The fact and the anxiety for the second outbreak are the research of COVID19 has brought great attention. Research on the effective and scientific prevention and warning of the second outbreak for all governments is urgent.

In this paper, we propose a compartment model based on SIHR and introduce first-order differential equations for cumulatively confirmed cases and death cases. Being different from previous articles, we use the Sigmoid function as the parameter expression reflecting the cumulative effect for the diagnosis rate, cure rate and mortality rate in the model. At the same time, we design the parameters in the model as a piecewise function at the lockdown time. To make the fitting data more accurate, we select eight sets of data in India and used the least squares optimizing method to carry out piecewise fitting, including the cumulative number of confirmed cases, existing cases, cured cases and death cases and their respective daily increase number. Based on the model and the parameters obtained from the fitting, we study the trend of the epidemic in India after the relaxation of control measures before and after the inflection point. In addition, the effect of medical quarantine capacity and the degree of cooperation of the people on the second outbreak of the epidemic are also studied. The model and research methods we use are not only applicable to India, but also provide a reference for other countries.

This paper is mainly composed of five sections. In Sect. 2, a SIHR-based compartmental model and fitted the parameters in the model is formulated. The impact of Indian relaxation of control measures before and after the inflection point on the future development trend of COVID-19 is analyzed in Sect. 3. Section 4 studies the impact of medical quarantine capacity and public cooperation on the second peak after relaxing control measures to prevent a second outbreak. Finally, in Sect. 5, we summarize the full text.

\section{Modeling and fitting}

\subsection{Mathematical model}

In 1927, Scottish epidemiologist Kermack and McKendrick proposed the famous SIR dynamic model for studying the epidemic laws of black death and plague [18]. This model and its improved model have been widely used in modeling and analysis of various infectious diseases. According to the transmission characteristics of COVID-19, we attempt to find a reliable formula to improve a SIHR-based compartmental model to investigate the possible consequences after relaxing control measures in India.

At any time $t$, the total population $N$ is divided into five categories: susceptible individuals $S(t)$, infected individuals $I(t)$ who are infectious but not diagnosed (including infectious persons during incubation period), hospitalized individuals $H(t)$ who have been diagnosed and completely isolated, namely existing confirmed cases, recovered individuals $R(t)$ and fatal individuals $D(t)$. So, we have the expression $N=S(t)+I(t)+H(t)+R(t)+D(t)$. Besides, we add cumulative confirmed cases $C(t)$ expressed by $C(t)=H(t)+R(t)+D(t)$. In our model, we have the following assumptions: The entire system is a closed system, regardless of the movement of personnel; all infected people can only be infected through human-to-human transmission; the recovered cases are entirely immune to the SARS-CoV-2, no longer infected. Based on the above assumptions, we present a schematic diagram of the model in Fig. 1.

As indicated by the arrow in Fig. 1, some susceptible population $S$ becomes infectious $I$ by contacting the infector, in other words, they move to $I$, and its probability is mainly determined by the transmission rate $\alpha(t)$ of the virus and the number of 


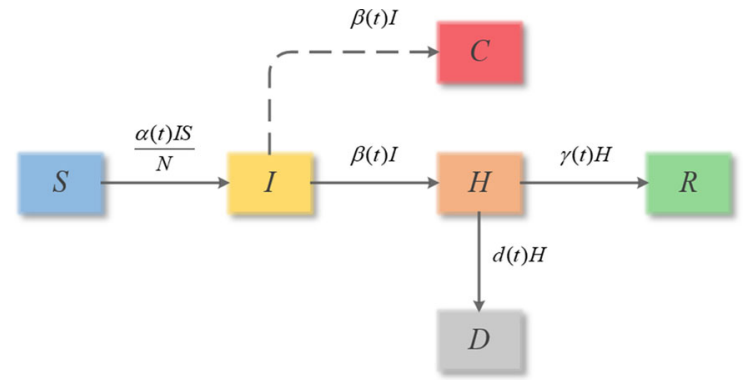

Fig. 1 Flow diagram of COVID-19 transmission

infectors. This process is reflected in the first subequation of Eq. (1). Similarly, the decrease in susceptible populations is accompanied by an increase in the number of infectious individuals, and the number of infectious $I$ decreases with the diagnosis rate $\beta(t)$ (inflow $H$ or $C$ ). This change is indicated in the second sub-equation. We assume that the diagnosed infectious individual will be sent to the hospital for treatment as soon as the diagnosis is confirmed, and the hospitalized individuals $H$ will be isolated in the hospital, so they will not infect the susceptible. Existing patients in the hospital will be healed or die at the rate of $\gamma(t)$ and $d(t)$ decrease, as shown in the third sub-equation. Meanwhile, the number of recovered $R$ and fatal individuals $D$ will increase at the corresponding rate. The cumulative confirmed cases, i.e., $C(t)=H(t)+R(t)+D(t)$, are expressed in the sixth sub-equation. Therefore, based on the six-state variables in Fig. 1, we use six firstorder differential equations to build an autonomous system, which is expressed by Eq. (1).

$$
\left\{\begin{array}{l}
\frac{\mathrm{d} S}{\mathrm{~d} t}=-\frac{\alpha(t) I S}{N} \\
\frac{\mathrm{d} I}{\mathrm{~d} t}=\frac{\alpha(t) I S}{N}-\beta(t) I \\
\frac{\mathrm{d} H}{\mathrm{~d} t}=\beta(t) I-(\gamma(t)+d(t)) H \\
\frac{\mathrm{d} R}{\mathrm{~d} t}=\gamma(t) H \\
\frac{\mathrm{d} D}{\mathrm{~d} t}=d(t) H \\
\frac{\mathrm{d} C}{\mathrm{~d} t}=\beta(t) I
\end{array}\right.
$$

where $\alpha(t), \beta(t), \gamma(t)$ and $d(t)$ represent the modelbased transmission rate, diagnosis rate, cure rate and mortality, respectively.

\subsection{Parameter description}

Note that the parameters $\alpha(t), \beta(t), \gamma(t)$ and $d(t)$ used in our model are all functions of time. In the literature, they are fixed. However, in practice, the state variables in the model are cumulative numbers, such as susceptible individuals, hospitalized individuals, cured cases and death cases, so their corresponding parameters should also be functions reflecting the cumulative effects. Therefore, we design the diagnosis rate $\beta(t)$, cure rate $\gamma(t)$ and mortality rate $d(t)$ as Sigmoid functions with cumulative effects like a distributive probability function within $[0,1]$.

Besides, we have also noticed that in the early stages of the outbreak, lots of countries have adopted measures such as city lockdown and testing, which can significantly reduce the spread of the virus and also help to confirm the diagnosis of infected people. The effects of the blockaded city on $\alpha(t), \beta(t), \gamma(t)$ and $d(t)$ are relatively significant, so we finally choose the piecewise function as the parameter in the model to distinguish stages before lockdown, after lockdown and relaxing.

The transmission rate $\alpha(t)$ directly determines the probability of infection of susceptible people. We know that isolation can cut off the spread of the virus to protect susceptible individuals. Compared with the mandatory isolation policies issued by governments such as lockdown, general isolation measures have little effect on the transmission rate so that the transmission rate can be estimated as a constant during a time interval. In addition, the relaxation of control measures will increase the probability of person-to-person contact, and the impact on the transmission rate is also significant. Therefore, $\alpha(t)$ is designed as

$\alpha(t)= \begin{cases}\alpha_{u}, & 0 \leq t<t_{\text {lock }}, \\ \alpha_{l}, & t_{\text {lock }} \leq t<t_{\text {relax }}, \\ \alpha_{r}, & t \geq t_{\text {relax }},\end{cases}$

where $t_{\text {lock }}$ and $t_{\text {relax }}$ represent the blocking time and relaxing time, $\alpha_{u}, \alpha_{l}$ and $\alpha_{r}$ represent the transmission rate in the periods of unlocking, locking and relaxing.

$\beta(t), \gamma(t)$ and $d(t)$ are designed as Sigmoid cumulative functions before and after the lockdown. When the relaxation policy is adopted, the diagnosis ability and the healing ability already have reached a 
certain level. If the degree of relaxation of the progressive relaxation policy is not high, and it has little effect on the ability of diagnosis and cure. Therefore, we believe that adopting a relaxation policy only has a direct impact on $\alpha(t)$, and has little impact on $\beta(t), \gamma(t)$ and $d(t)$. We assume that $\beta(t)$, $\gamma(t)$ and $d(t)$ have the same form of function expression in the lockdown period and after relaxing control. The diagnosis rate $\beta(t)$ is a cumulative function with time, so we design $\beta(t)$ as

$\beta(t)= \begin{cases}\frac{1}{1+e^{-k_{\beta u}\left(t-\tau_{\beta u}\right)}}, & 0 \leq t<t_{\text {lock }}, \\ \frac{1}{1+e^{-k_{\beta l}\left(t-\tau_{\beta l}\right)},}, & t \geq t_{\text {lock }},\end{cases}$

where $k_{\beta u}, \tau_{\beta u}, k_{\beta l}$ and $\tau_{\beta l}$ represent the Sigmoid function parameters of the diagnosis rate before and after the lockdown, respectively. $k$ represents the rising slope of the $S$ function and the range is $(0,+\infty) ; \tau$ represents the delay time of the $S$ function and the range is $(-\infty,+\infty)$. Now, we choose the expression of $\beta(t)$ in the continuous-time period to illustrate the role of the Sigmoid function parameters. As shown in Fig. 2, when $\tau$ is a constant value, the higher the value of $k$, the more significant the slope rate, $\beta(t)$, is around $t=\tau$. Therefore, in the diagnosis rate $\beta(t)$, we can understand $k$ as a parameter describing the cumulative diagnosis speed. Figure $2 b$ shows the effect delay time $\tau$ in the diagnosis that is determined by the government's decision.

(a) $\tau=10$

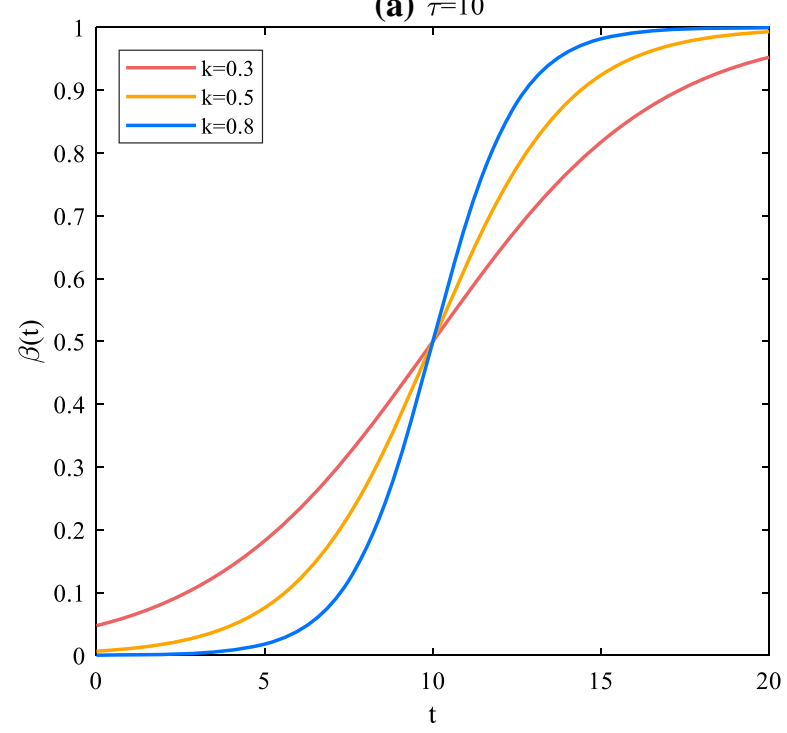

Fig. 2 Effect of Sigmoid function parameter changes on trends $\gamma(t)$ represents the cure rate of $H$ to $R$ in the model, and $d(t)$ the mortality rate from $H$ to $D$. With the increase in time, $\gamma(t)$ increases as cumulative function. Conversely, $d(t)$ decreases. Both are given by Eqs. (4) and (5).

$\gamma(t)= \begin{cases}\frac{1}{1+e^{-k_{\gamma / u}\left(t-\tau_{j u}\right)}}, & 0 \leq t<t_{\text {lock }}, \\ \frac{1}{1+e^{-k_{\gamma l} l\left(t-\tau_{\gamma / l}\right)}}, & t \geq t_{\text {lock }},\end{cases}$

$d(t)= \begin{cases}\frac{1}{\left.1+e^{k} d u^{(t-\tau} d u\right)}, & 0 \leq t<t_{\text {lock }}, \\ \frac{1}{1+e^{d_{d l}(t-\tau} \tau^{(t)}}, & t \geq t_{\text {lock }},\end{cases}$

among them, the range of $k_{\gamma u}, k_{\gamma l}, k_{d u}$ and $k_{d l}$ is $(0,+\infty), \tau_{\gamma u}, \tau_{\gamma l}, \tau_{d u}$ and $\tau_{d l}$ is $(-\infty,+\infty)$. Note that Eq. (4) is monotonically increasing form, but Eq. (5) is monotonically decreasing form.

\subsection{Data fitting}

The web of [7] in this study is the primary, and detailed data source of COVID-19 for the recovered cases have not been reported in WHO [2].

Here, we use the least square functions fmincon and lsqnonlin of MATLAB to fit the parameters of $\alpha(t), \beta(t), \gamma(t)$ and $d(t)$ in the model. Eight sets of data from March 1 to June 7 in India are selected as fitting data. The objective function minimized by least squares is described as

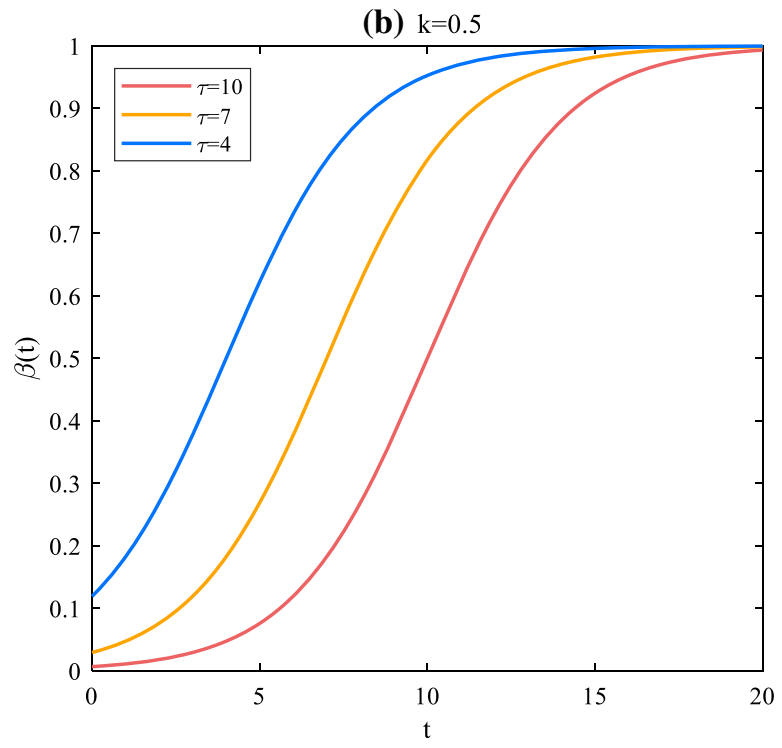




$$
\begin{aligned}
f(\boldsymbol{k}, t)= & \sum_{i=1}^{t}(C(t)-\hat{C}(t))+\sum_{i=1}^{t}(H(t)-\hat{H}(t)) \\
& +\sum_{i=1}^{t}(R(t)-\hat{R}(t))+\sum_{i=1}^{t}(D(t)-\hat{D}(t)) \\
& +\sum_{i=1}^{t}(\Delta C(t)-\Delta \hat{C}(t))+\sum_{i=1}^{t}(\Delta H(t)-\Delta \hat{H}(t)) \\
& +\sum_{i=1}^{t}(\Delta R(t)-\Delta \hat{R}(t))+\sum_{i=1}^{t}(\Delta D(t)-\Delta \hat{D}(t)),
\end{aligned}
$$

where $\boldsymbol{k}$ represents the parameter vector to be fitted, $C(t), H(t), R(t)$ and $D(t)$ represent the cumulative confirmed cases, existing cases, cured cases and death cases calculated by Eq. (1), and $\hat{C}(t), \hat{H}(t), \hat{R}(t)$ and $\hat{D}(t)$ are the actual reported data. $\Delta C(t), \Delta H(t)$, $\Delta R(t), \Delta D(t), \Delta \hat{C}(t), \Delta \hat{H}(t), \Delta \hat{R}(t)$ and $\Delta \hat{D}(t)$, respectively, indicate the corresponding newly added cases or daily cases, which is the difference between the cumulative number of cases in two consecutive days.

In data fitting, we need to use the initial value of each state variable, where $C(0), H(0), R(0)$ and $D(0)$ can be obtained by reporting data. The initial value of $I$ can be estimated by $\sigma H(0)$, where $\sigma=5.1$ is expressed as the incubation period [19]. So $S(0)=N-I(0)-C(0)$, where $N$ is 1.324 billion was obtained from the UNFPA website [20]. Considering the impact of the lockdown on $\alpha(t), \beta(t), \gamma(t)$ and $d(t)$, we divide the data from March 1 to June 7 in India into two parts before and after the blockade to fit. According to reports, the Indian government began implementing a nationwide lockdown plan on March 25 [8]. The coefficients based on the data fitting are given in Table 1, and the fitting curve and the data scatter plot are given in Fig. 3.

To evaluate the goodness of the fitting results, we introduce the coefficient of determination $R^{2}$, the closer the value of $R^{2}$ is to 1 , indicating the better the fit between the fitted value and the observed value; otherwise, the fitting degree is worse. Generally, the coefficient of determination $R^{2}$ can be expressed as
Table 1 Fitting results of coefficients

\begin{tabular}{llrl}
\hline Coefficient & Range & \multicolumn{1}{c}{ Value } & Source \\
\hline$\alpha_{u}$ & $(0,1)$ & 0.2909 & Fitted \\
$k_{\beta u}$ & $(0,+\infty)$ & 0.0011 & Fitted \\
$\tau_{\beta u}$ & $(-\infty,+\infty)$ & 1844.2306 & Fitted \\
$k_{\gamma u}$ & $(0,+\infty)$ & 0.0507 & Fitted \\
$\tau_{\gamma u}$ & $(-\infty,+\infty)$ & 163.3708 & Fitted \\
$k_{d u}$ & $(0,+\infty)$ & 0.0282 & Fitted \\
$\tau_{d u}$ & $(-\infty,+\infty)$ & -246.0921 & Fitted \\
$\alpha_{l}$ & $(0,1)$ & 0.13830 & Fitted \\
$k_{\beta l}$ & $(0,+\infty)$ & 0.00754 & Fitted \\
$\tau_{\beta l}$ & $(-\infty,+\infty)$ & 338.00666 & Fitted \\
$k_{\gamma l}$ & $(0,+\infty)$ & 0.01330 & Fitted \\
$\tau_{\gamma l}$ & $(-\infty,+\infty)$ & 286.40878 & Fitted \\
$k_{d l}$ & $(0,+\infty)$ & 0.00590 & Fitted \\
$\tau_{d l}$ & $(-\infty,+\infty)$ & -954.98765 & Fitted \\
\hline
\end{tabular}

$$
\begin{aligned}
R^{2} & =\frac{E_{\mathrm{SS}}}{T_{\mathrm{SS}}}=1-\frac{R_{\mathrm{SS}}}{T_{\mathrm{SS}}}, \\
& =\frac{\sum_{i=1}^{n}\left(\hat{y}_{i}-\bar{y}\right)^{2}}{\sum_{i=1}^{n}\left(y_{i}-\bar{y}\right)^{2}}=1-\frac{\sum_{i=1}^{n}\left(y_{i}-\hat{y}_{i}\right)^{2}}{\sum_{i=1}^{n}\left(y_{i}-\bar{y}\right)^{2}},
\end{aligned}
$$

among them, $T_{\mathrm{SS}}$ is the total sum of squares, $E_{\mathrm{SS}}$ the explained sum of squares, $R_{\mathrm{SS}}$ the residual sum of squares, $y_{i}$ the observed value of real data, $\bar{y}$ the average value of real data observations, $\hat{y}_{i}$ the fitted value.

To compare with the Sigmoid function used, we also set $\alpha(t), \beta(t), \gamma(t)$ and $d(t)$ as constant parameters for fitting. The fitting curve results can also track the trend of the actual data, but the fitting degree is not as high as when using the Sigmoid function. From Table 2, the goodness of fit of the cumulative and daily confirmed cases between the $S$ function and the constant parameters before lockdown is not much different. We believe that India's diagnosis rate did not change much before lockdown, and it was an approximately constant value. The values $R^{2}$ of the cured cases and fatal cases before the blockade are relatively low, because there are few cured cases and fatal cases in the early stage of the outbreak, resulting in no apparent trend. After the lockdown, it can be noticed that the $R^{2}$ fitted by the Sigmoid function is generally larger than that 
(a)

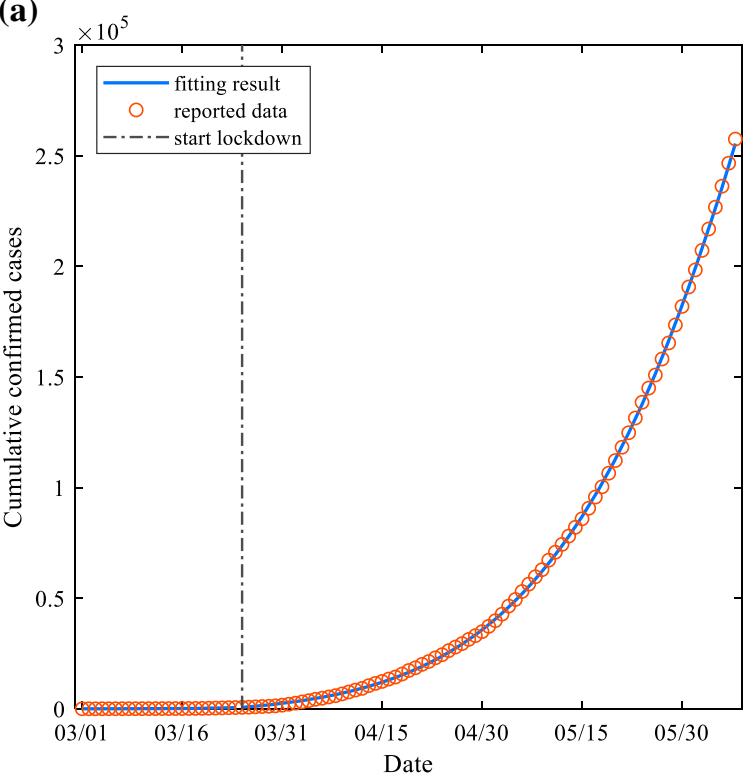

(c)

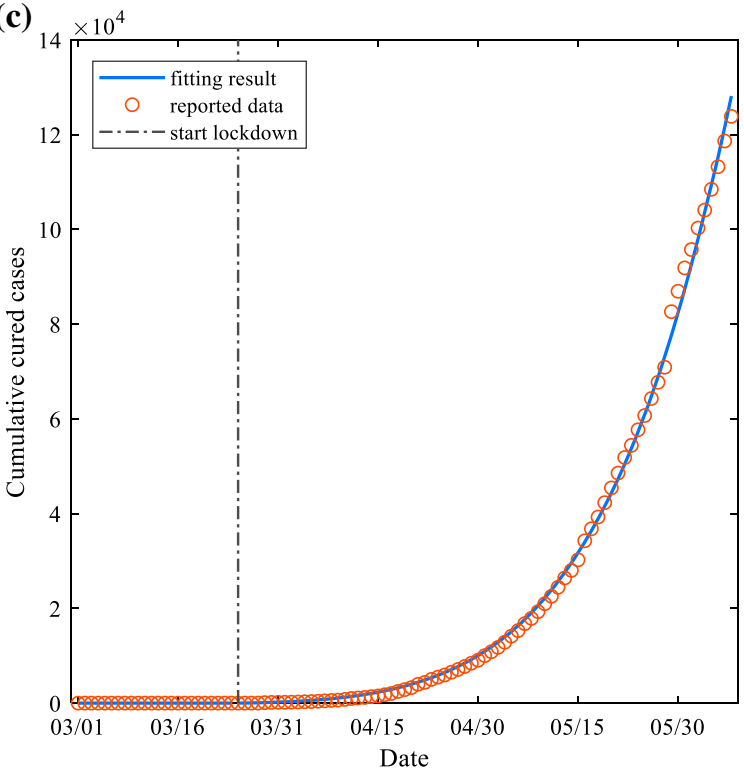

(b)

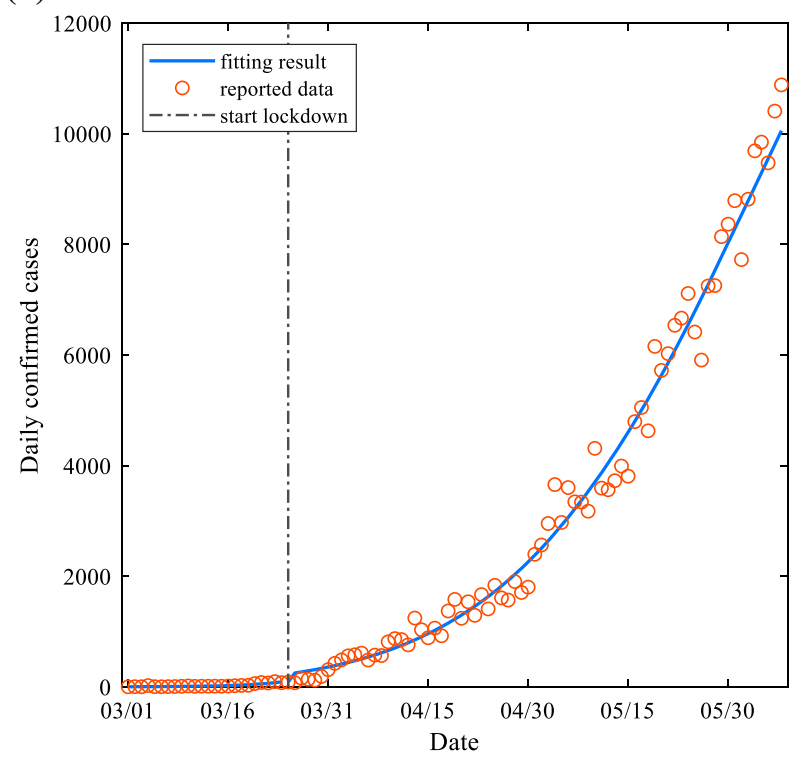

(d)

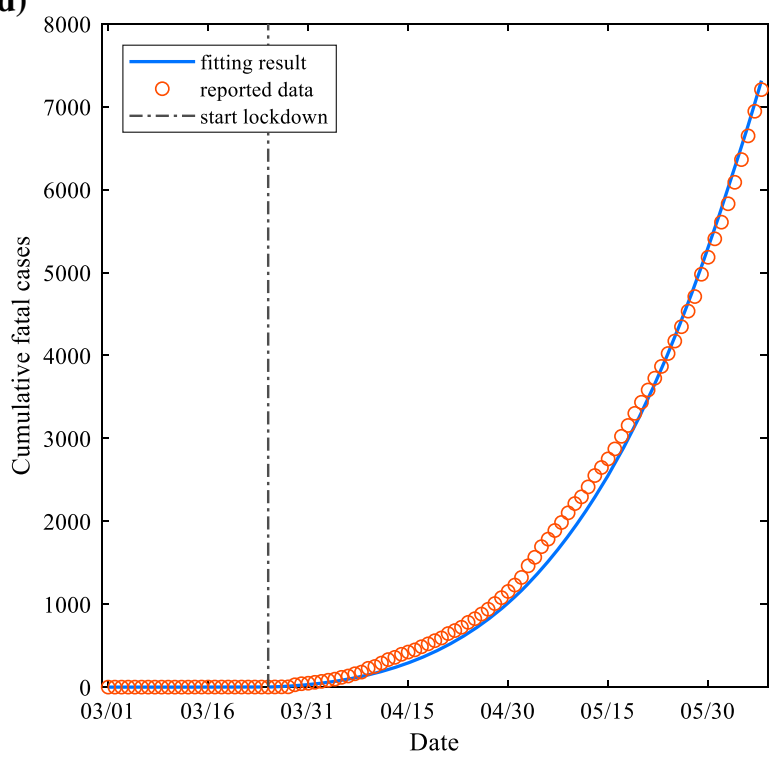

Fig. 3 Model-based fitting curve (solid blue line) and data scatter plot (red circle), where a refers to cumulative confirmed cases, b daily confirmed cases, $\mathbf{c}$ cumulative cured cases and $\mathbf{d}$ cumulative fatal cases

obtained by constant parameters. It further illustrates the superiority of using the Sigmoid function as a parameter in the model.

\section{Impact of relaxing control in India}

According to the trend of daily confirmed cases, we roughly divide the development of the entire epidemic into four stages.

Stage I: Initial stage. Initially, fewer cases are diagnosed, and there is an upward trend. 
Table 2 Goodness of fit under different types of model parameters

\begin{tabular}{|c|c|c|c|c|c|c|c|c|}
\hline \multirow[t]{3}{*}{ Type of model parameters } & \multicolumn{8}{|c|}{ The value of $R^{2}$} \\
\hline & \multicolumn{4}{|c|}{ Before lockdown (March 1 to March 24) } & \multicolumn{4}{|c|}{ After lockdown (March 25 to June 7) } \\
\hline & C & $\Delta C$ & $R$ & $D$ & $C$ & $\Delta C$ & $R$ & $D$ \\
\hline Sigmoid function & 0.9902 & 0.8811 & 0.7841 & 0.5441 & 0.9999 & 0.9851 & 0.9983 & 0.9949 \\
\hline Constant parameter & 0.9903 & 0.8809 & 0.6954 & 0.4904 & 0.9987 & 0.9736 & 0.9838 & 0.9855 \\
\hline
\end{tabular}

Stage II: Outbreak stage. The virus has accelerated and spread widely, and the number of confirmed cases has multiplied.

Stage III: Peak stage. The number of daily confirmed cases reach a peak, appear inflection point and show a downward trend.

Stage IV: Rehabilitation stage. The number of newly confirmed cases has continued to decline, and the epidemic situation can be controlled basically.

Figure 4 shows the current stage of some countries. We can see that China has entered the rehabilitation stage very early; most European countries have also entered the rehabilitation stage, and the economy has begun to recover during the prevention and control of the epidemic; the current severe epidemic in the USA is still at the peak stage; Brazil, which is also relatively serious, is at the outbreak stage of a rapidly growing case.

It is noted that India is currently still in an outbreak stage. According to the daily confirmed case data from [7], it can be seen that the transmission has not yet reached the inflection point of the pandemic, and the newly confirmed cases still show a continuous upward trend. However, the Indian government announced on May 30 that while continuing to extend the lockdown period, some areas with lighter epidemics would plan to reopen religious venues, hotels and restaurants on June 8. Now we use our model and fit parameters to estimate the development of the epidemic after different degrees of relaxation in India.

\subsection{Relaxing control before the inflection point in India}

We assume that the degree of government deregulation is positively correlated with the degree of increase in the transmission rate in the model; in other words, if the degree of relaxation implemented by the government after the lockdown is $n \%$ of the lockdown period, then the transmission rate $\alpha(t)$ will

Fig. 4 Stage of epidemic situation in some countries

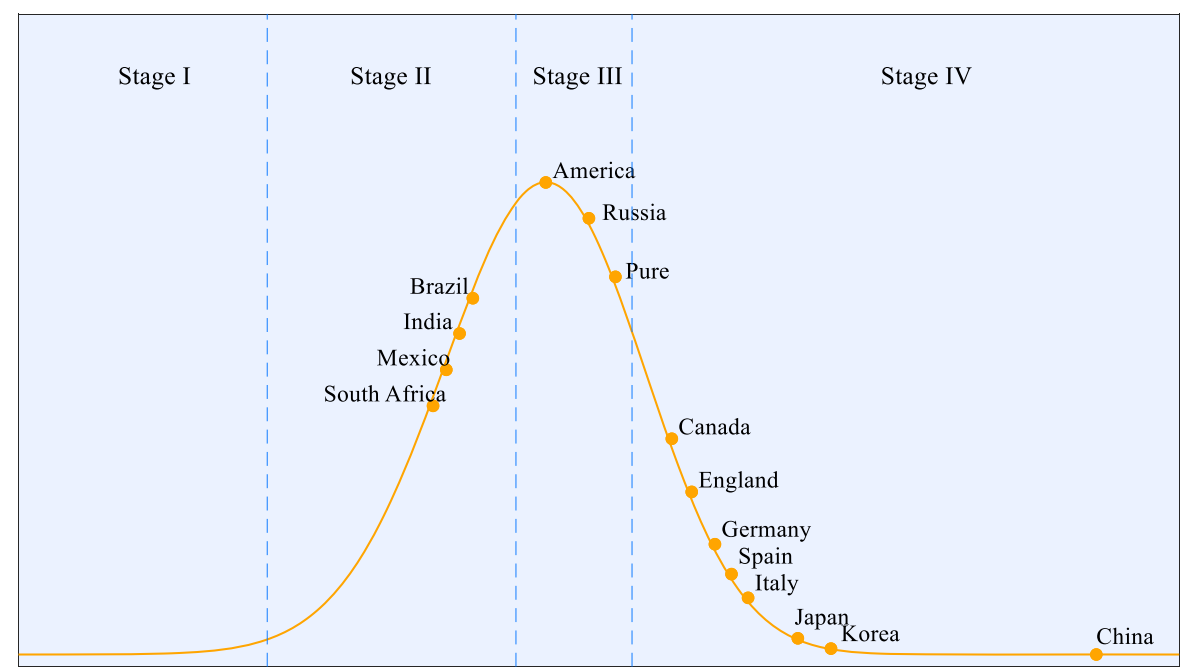


increase by $\left(\alpha_{u}-\alpha_{l}\right) \times n \%$ on the basis of the lockdown period, so the transmission rate of relaxation is $\alpha_{r}=\alpha_{l}+\left(\alpha_{u}-\alpha_{l}\right) \times n \%$. According to our fitting results, India's newly confirmed case curve on June 8 has not yet reached the inflection point, and it is still in Stage II. There are many contagious infections in the crowd, so we assume that the degree of relaxation control is set to be lower, $5 \%$ and $10 \%$, respectively.

Figure 5 shows the comparison curves of newly confirmed cases, cumulative confirmed cases and cumulative fatal cases with $0 \%, 5 \%$ and $10 \%$ relaxation on June 8 , respectively. According to Fig. 5a, as the degree of relaxation increases, the peak

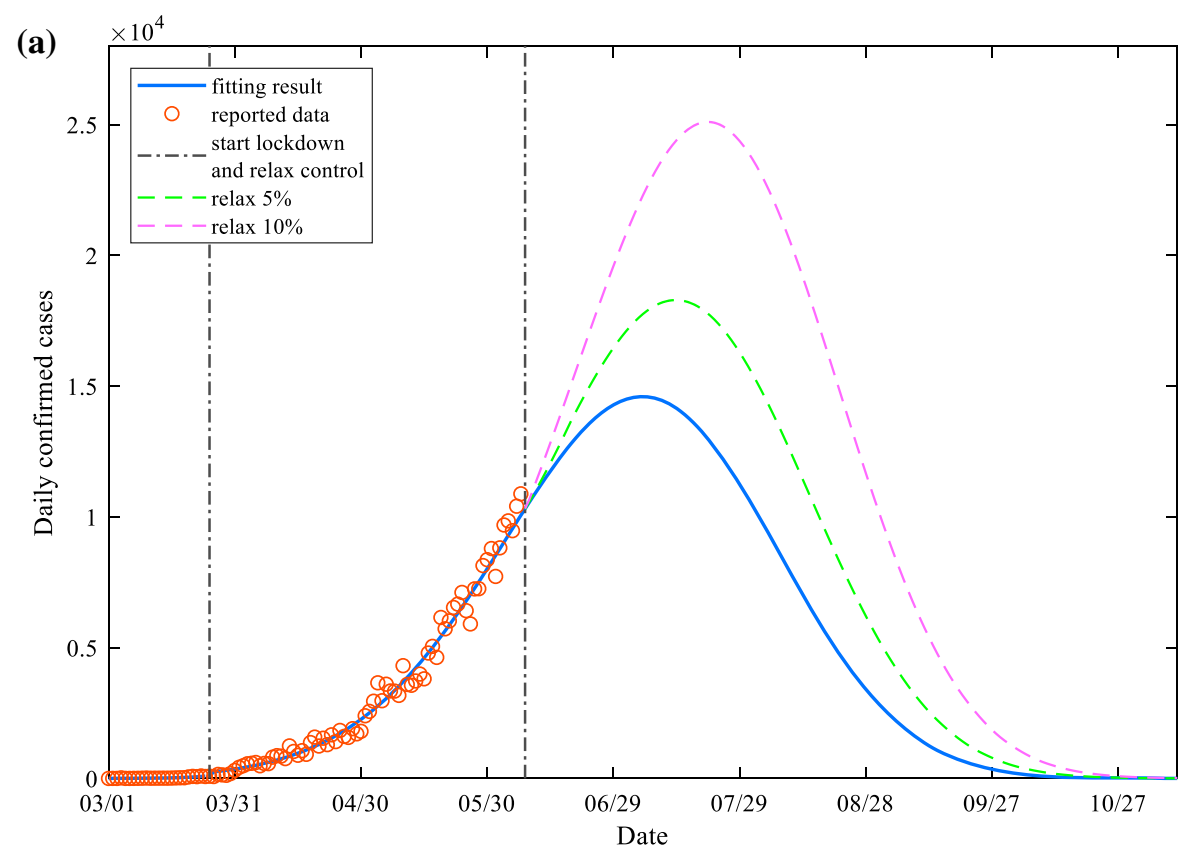

(b)

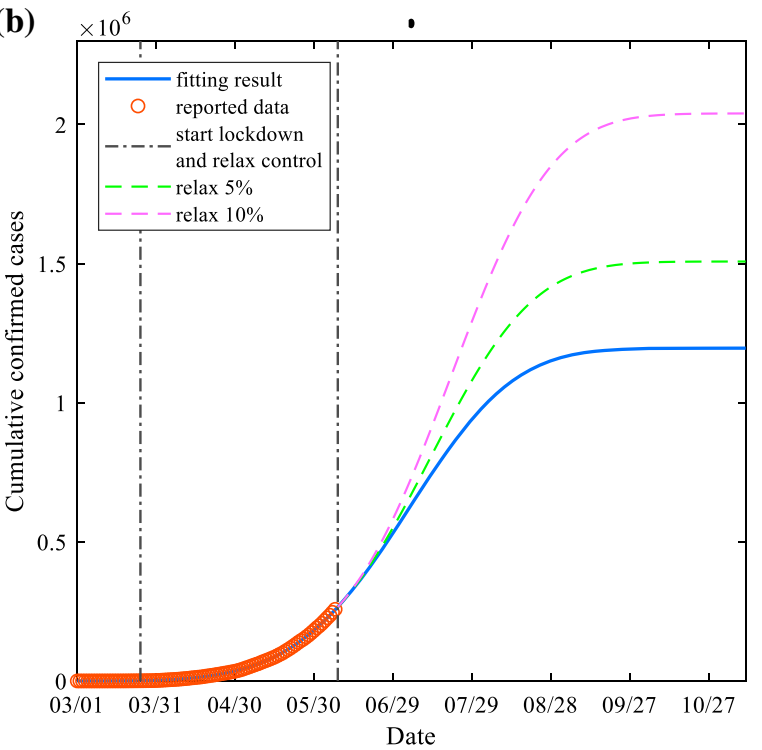

(c)

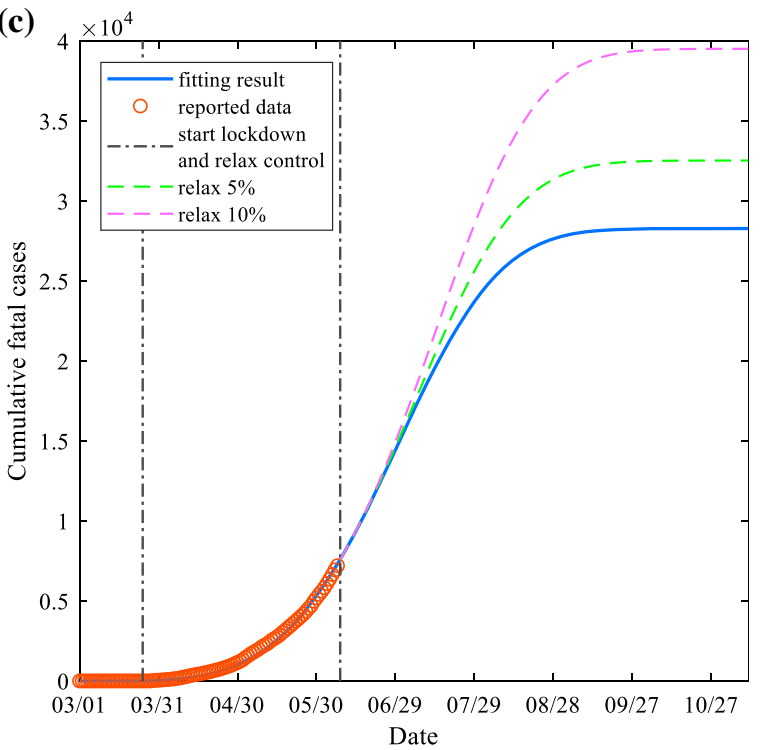

Fig. 5 Impact of India's first relaxation of controls on June 8, a daily confirmed cases, b cumulative confirmed cases and c cumulative fatal cases. A slight relaxation of control before the inflection point will cause more serious consequences 
date of the newly confirmed case curve is postponed, and the date to reach the peak is July $6(0 \%)$, July 14 $(5 \%)$ and July $21(10 \%)$, respectively. The peak value also increases with the degree of deregulation, and the specific peak value can reach 14,595 cases $(0 \%)$, 18,288 cases $(5 \%)$ and 25,096 cases (10\%). Compared with not adopting a relaxation policy, a $5 \%$ increase in relaxation results in a peak increase of approximately $25.30 \%$; an increase of $10 \%$ on the basis of the lockdown period results in a peak increase of approximately $71.95 \%$. At the same time, Fig. $5 \mathrm{~b}$ and $\mathrm{c}$ also shows the trend of the cumulatively confirmed cases and cumulative death cases under different degrees of relaxation. Our model predicts that without the relaxation policy, the cumulative number of confirmed cases eventually reaches above 1.196 million cases, and the cumulative number of deaths reaches above 28,280 cases. If $5 \%$ control is relaxed, these two values increase by $26.00 \%$ and $15.03 \%$, respectively; if $10 \%$ is relaxed, they increase by $70.57 \%$ and $39.71 \%$. The model also shows that if according to the current medical level and the public's consciousness, India's COVID-19 will ultimately end in November.

It can be seen that taking a relaxation policy before the inflection point will lead to many more confirmed and dead cases even if the relaxation rate is $5 \%$. However, due to increasing downward pressure on the economy, India had to implement a progressive relaxation policy on June 8.

\subsection{Relaxing control after the inflection point in India}

According to the data from June 8 to the present (June 22), we assume that the degree of relaxing policy adopted for the first time on June 8 is $5 \%$. On this basis, we analyze the impact of further deblocking on the subsequent development of India's COVID-19. According to the curve of 5\% relaxation in Fig. 5, in late August, the epidemic can be controlled, and the curve of newly diagnosed cases shows a rapid downward trend.

Now, we assume that the second relaxation policy is implemented on August 22, setting the relaxation levels to $30 \%, 45 \%$ and $60 \%$ on the basis of the lockdown period. Figure 6 depicts the comparison curves of newly confirmed cases, cumulative confirmed cases and fatal cases for different degrees of relaxation $(30 \%, 45 \%$ and $60 \%)$ after further relaxation on August 22. In Fig. 6a, after further relaxing the control, as the degree of unblocking increases, the downward trend of the curve of the newly diagnosed case slows down, and even a secondary peak may occur, which in turn leads to a secondary outbreak of the epidemic. If no further relaxation policy is adopted, the curve of daily diagnosed cases will show a rapid downward trend after August 22, and eventually, there will be zero new cases after 89 days. After the relaxation policy is adopted, this value will become 111 days and 127 days and 144 days, an increase in the duration time of $24.72 \%, 42.70 \%$ and $61.80 \%$ will be caused, respectively. At the same time, Fig. $6 \mathrm{~b}$ and $\mathrm{c}$ also shows that as the degree of further relaxation increases, the final stable value of the cumulative confirmed cases and fatal cases will relatively increase, and the detailed data comparison of Fig. 6 is given by Table 3. (In Fig. 6c, we can see that the real data after June 17 are slightly higher than the fitted curve, which is caused by the sudden increase of more than 2000 new deaths on June 17. There is a similar situation in the subsequent figures.)

These results show that although the deterioration of the epidemic caused by the relaxation policy after the inflection point is not as serious as before the inflection point, the higher the degree of relaxation, the more likely a secondary outbreak will occur. This will not only extend the duration of the epidemic, but also lead to more confirmed cases and deaths, which will put more pressure on the health care system.

Now, we choose a further relaxation degree of $60 \%$ and set the dates of the second relaxation policy to August 15, August 22 and August 29 to study the impact of the relaxation policy on the epidemic at different time points after the inflection point. It can be seen from Fig. 7 that although the relaxation of control at different time points has little effect on the duration of the epidemic, the more relaxed the control in advance, the higher peak value of the second peak of daily confirmed cases, and even more than the primary peak. At the same time, the cumulative confirmed cases and cumulative fatal cases will also increase significantly.

Note that under the same degree of relaxation, premature measures may lead to a more secondary severe outbreak. If a further relaxation policy is adopted shortly after the peak period, there will still be a large number of infectors who have not been 


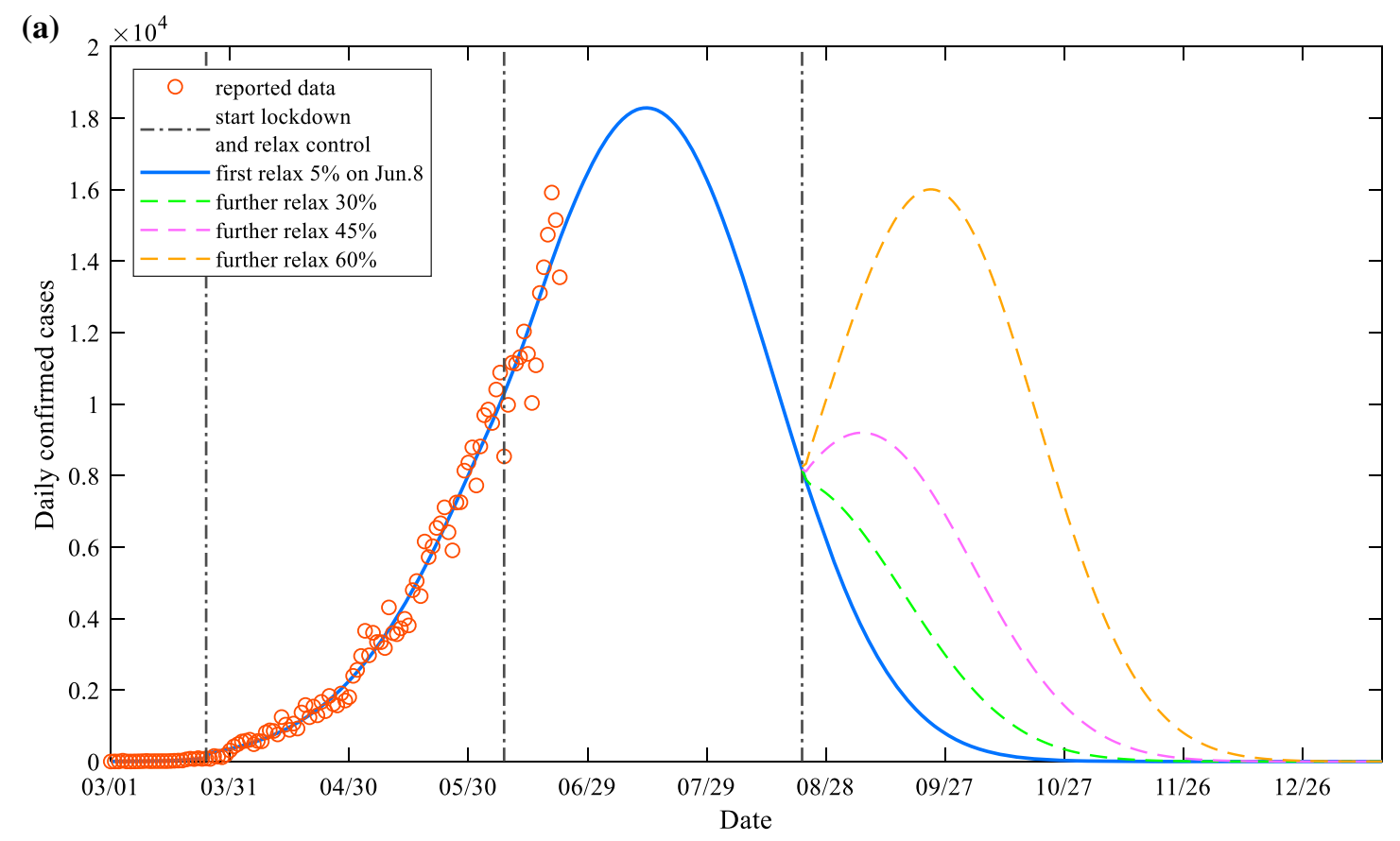

(b)

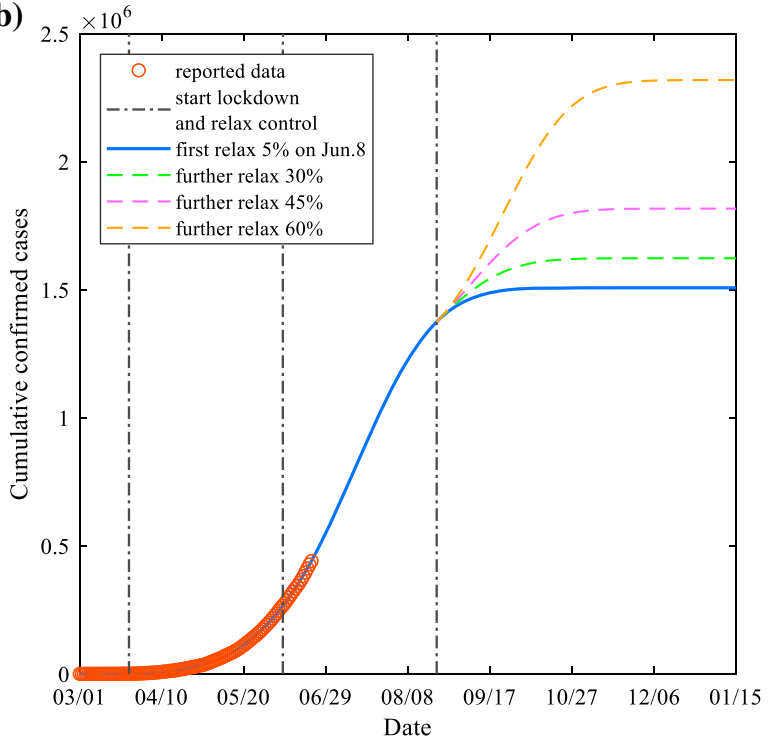

Fig. 6 Impact of India on, a daily confirmed cases, b cumulative confirmed cases and c cumulative fatal cases, after further relaxation of control on August 22. After the turning

identified, resulting in more susceptible people being infected. Therefore, we suggest that if the economy tolerance allows, delaying further relaxation after the inflection point and reducing the degree of relaxation will make the epidemic more controllable.

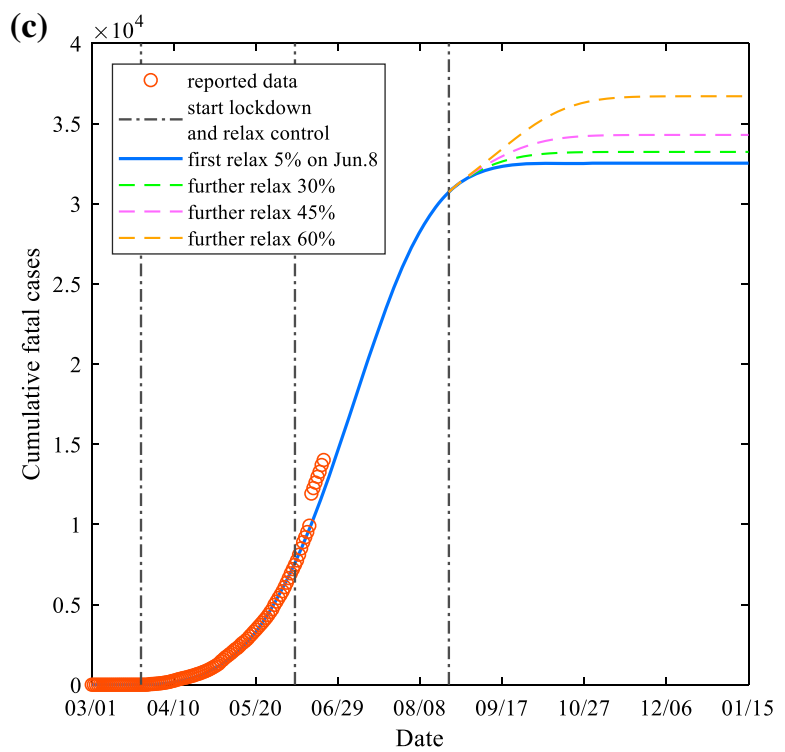

point, as the degree of relaxation increased, a second outbreak of the epidemic occurred, resulting in more confirmed cases and deaths

\section{Prevention of a possible second outbreak}

It can be concluded in the previous section that when the relaxation policy is adopted after the inflection point, under the relaxation to some extent, it will lead 
Table 3 Comparison of data after further relaxing control at different levels on August 22

\begin{tabular}{|c|c|c|c|c|c|}
\hline \multirow{2}{*}{$\begin{array}{l}\text { Degree of further } \\
\text { relaxation }(\%)\end{array}$} & \multicolumn{3}{|c|}{ Daily confirmed cases curve } & \multirow{2}{*}{$\begin{array}{l}\text { Cumulative confirmed } \\
\text { cases curve } \\
\text { Stable value (increase } \\
\text { rate compared to } 0 \% \text { ) }\end{array}$} & \multirow{2}{*}{$\begin{array}{l}\text { Cumulative fatal cases } \\
\text { curve } \\
\text { Stable value (increase } \\
\text { rate compared to } 0 \% \text { ) }\end{array}$} \\
\hline & $\begin{array}{l}\text { Second } \\
\text { peak value }\end{array}$ & $\begin{array}{l}\text { Second } \\
\text { peak time }\end{array}$ & $\begin{array}{l}\text { Duration of the outbreak } \\
\text { (increase rate compared to } 0 \% \text { ) }\end{array}$ & & \\
\hline 0 & - & - & $89(-)$ & $1,507,406(-)$ & $32,527(-)$ \\
\hline 30 & - & - & $111(24.72 \%)$ & $1,622,754(7.652 \%)$ & $33,228(2.155 \%)$ \\
\hline 45 & 9199 & 15 & $127(42.70 \%)$ & $1,816,494(20.50 \%)$ & $34,288(5.414 \%)$ \\
\hline 60 & 16,007 & 32 & $144(61.80 \%)$ & $2,318,547(53.81 \%)$ & $36,701(12.83 \%)$ \\
\hline
\end{tabular}

to a second outbreak of the epidemic. Relaxing control will inevitably lead to an increase in the transmission rate $\alpha(t)$ of the virus among the population, which in turn makes susceptible individuals more likely to be infected by the infectious person, increasing the number of infectious individuals $I$. To prevent the second outbreak, the government can improve the medical quarantine capacity after relaxing control, and then improve the diagnosis rate $\beta(t)$, so that more infectors can be detected and isolated. The degree of cooperation of the people after the relaxation of control is also essential for pandemic prevention and control. If the vast majority of the people can cooperate with the government's regulations, maintain a certain social distance and wear masks, the transmission rate $\alpha(t)$ will also be reduced. This section studies the impact of medical quarantine capacity and public cooperation on the second outbreak after relaxing control to provide some preventive measures for the Indian government after unblocking.

\subsection{Effect of medical quarantine ability} on the second outbreak

The medical quarantine capability can be expressed in the size of the detection range, the speed of the detection and the sufficiency of nucleic acid detection kit. When the supply of kits is sufficient, expanding the detection range and increasing the detection speed can enable the unidentified infectors in the crowd to be diagnosed and isolated as soon as possible, thereby reducing the possibility of susceptible individuals being infected. In our model, the diagnosis rate is directly related to the medical quarantine ability. The higher the medical quarantine ability, the higher the diagnosis rate. In the previous sections, we introduced the expression form of $\beta(t)$. Now, we update $\beta(t)$ to the following form to study the impact of medical quarantine capacity after further relaxation of control.

$\beta(t)= \begin{cases}\frac{1}{\left.1+e^{-k_{\beta u}(t-\tau} \tau_{\beta u}\right)}, & 0 \leq t<t_{\text {lock }}, \\ \frac{1}{\left.1+e^{-k_{\beta l}(t-\tau} \tau_{\beta l}\right)}, & t_{\text {lock }} \leq t<t_{\text {relax } 2}, \\ \frac{1}{\left.1+e^{-k_{\beta r}(t-\tau} \tau_{r r}\right)}, & t \geq t_{\text {relax } 2,}\end{cases}$

where $t_{\text {relax2 }}$ represents the moment of further relaxing the control measures after the inflection point.

We chose to take the further relaxation of $60 \%$ control as the benchmark on August 22 to study the impact of the different diagnosis rates caused by the medical quarantine capacity on the epidemic after a further relaxation of control. By changing the combination form of $k_{\beta r}$ and $\tau_{\beta r}$, the initial value and rising trend of the diagnosis rate $\beta(t)$ after unsealing can be changed. Figure 8 shows the diagnosis rate curves of different combinations of $k_{\beta r}$ and $\tau_{\beta r}$ after relaxing control. We use the diagnosis rate curve obtained by $k_{\beta l}$ and $\tau_{\beta l}$ during the blockade period as the baseline (solid blue line) and increase the diagnosis rate on this basis to obtain Curves I, II and III, respectively. In addition, we also reduced the diagnosis rate by $5.425 \%$ on the basis of the baseline to obtain Curve IV, to observe the impact of the medical quarantine level after deblocking is lower than the blockade period. According to Fig. 8, we can notice that the growing trends of the diagnosis rate Curves II, III and IV are similar to the growing trend of the baseline, but the initial value has changed. Curve I grows faster than other curves and becomes 


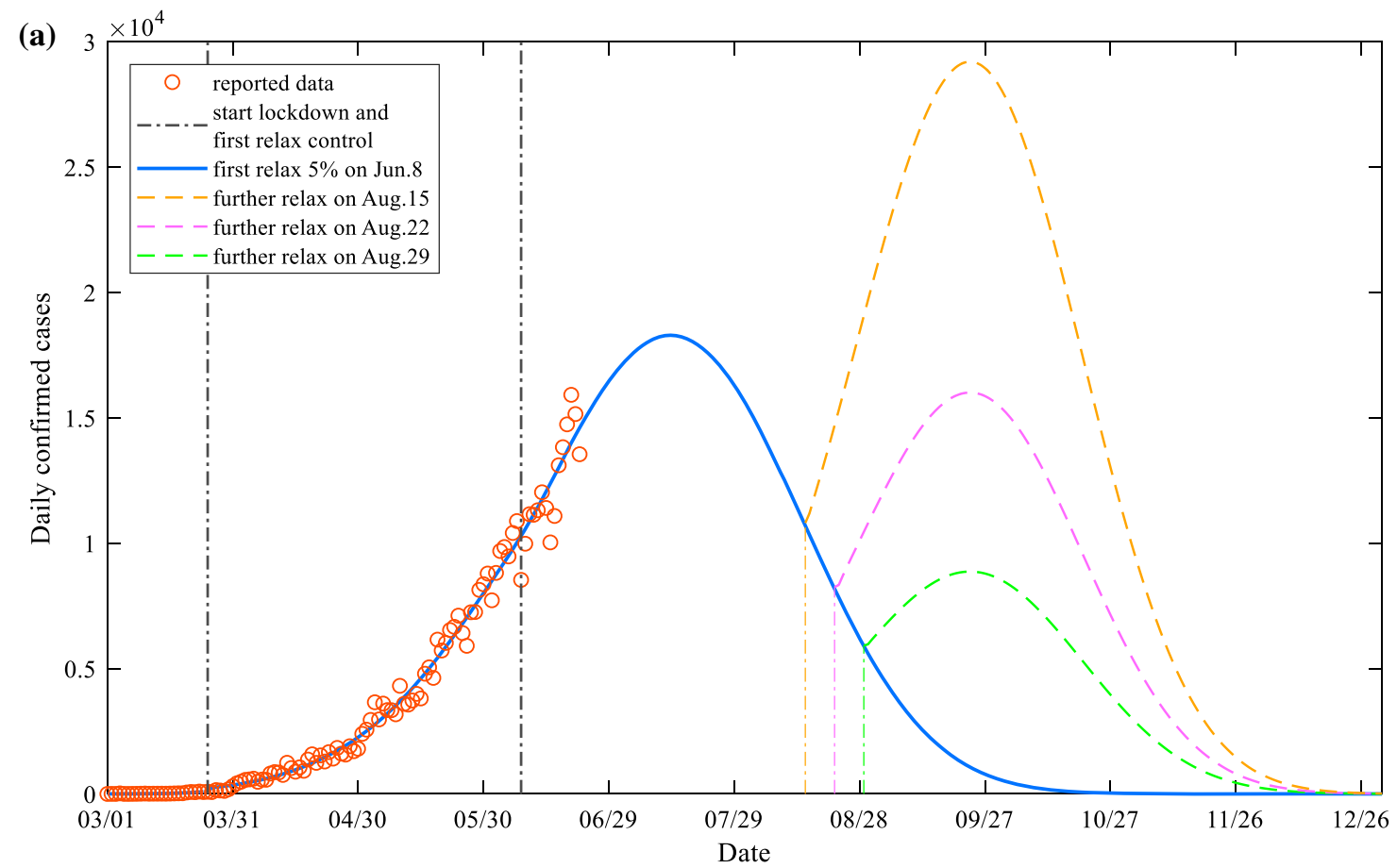

(b)

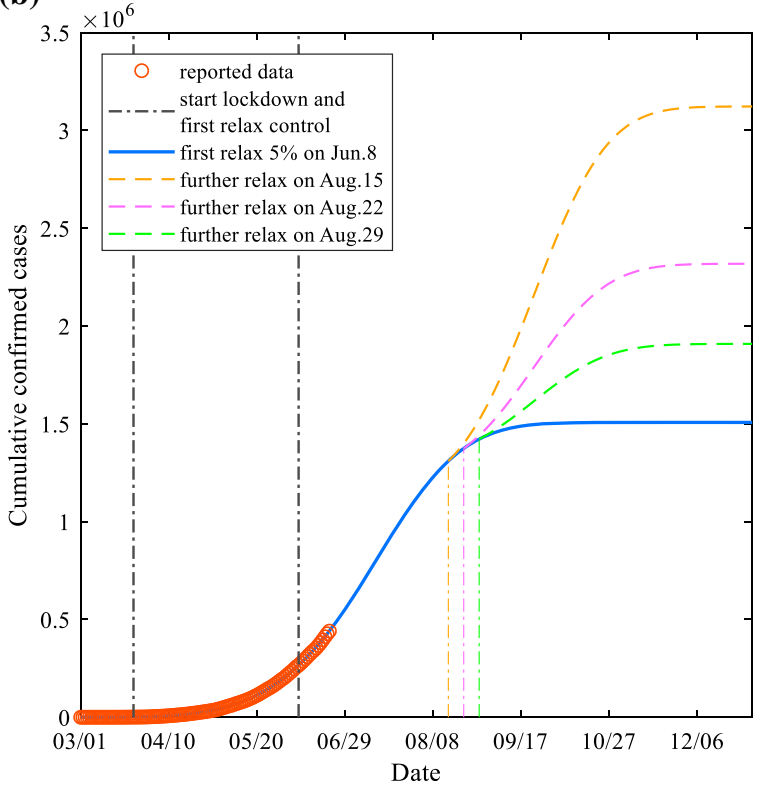

Fig. 7 Impact of the same degree of relaxation $(60 \%)$ on, a daily confirmed cases, b cumulative confirmed cases, c cumulative fatal cases after a further relaxation of control at different time points. Under the same degree of relaxation (c)

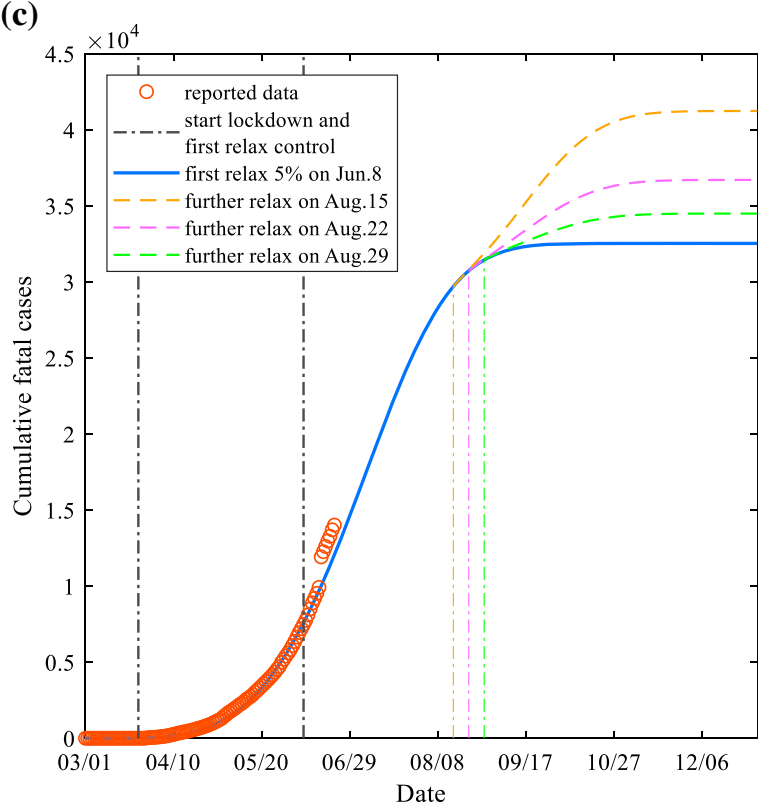

after the inflection point, the earlier the relaxation, the more likely to have a second outbreak even more than the first outbreak 


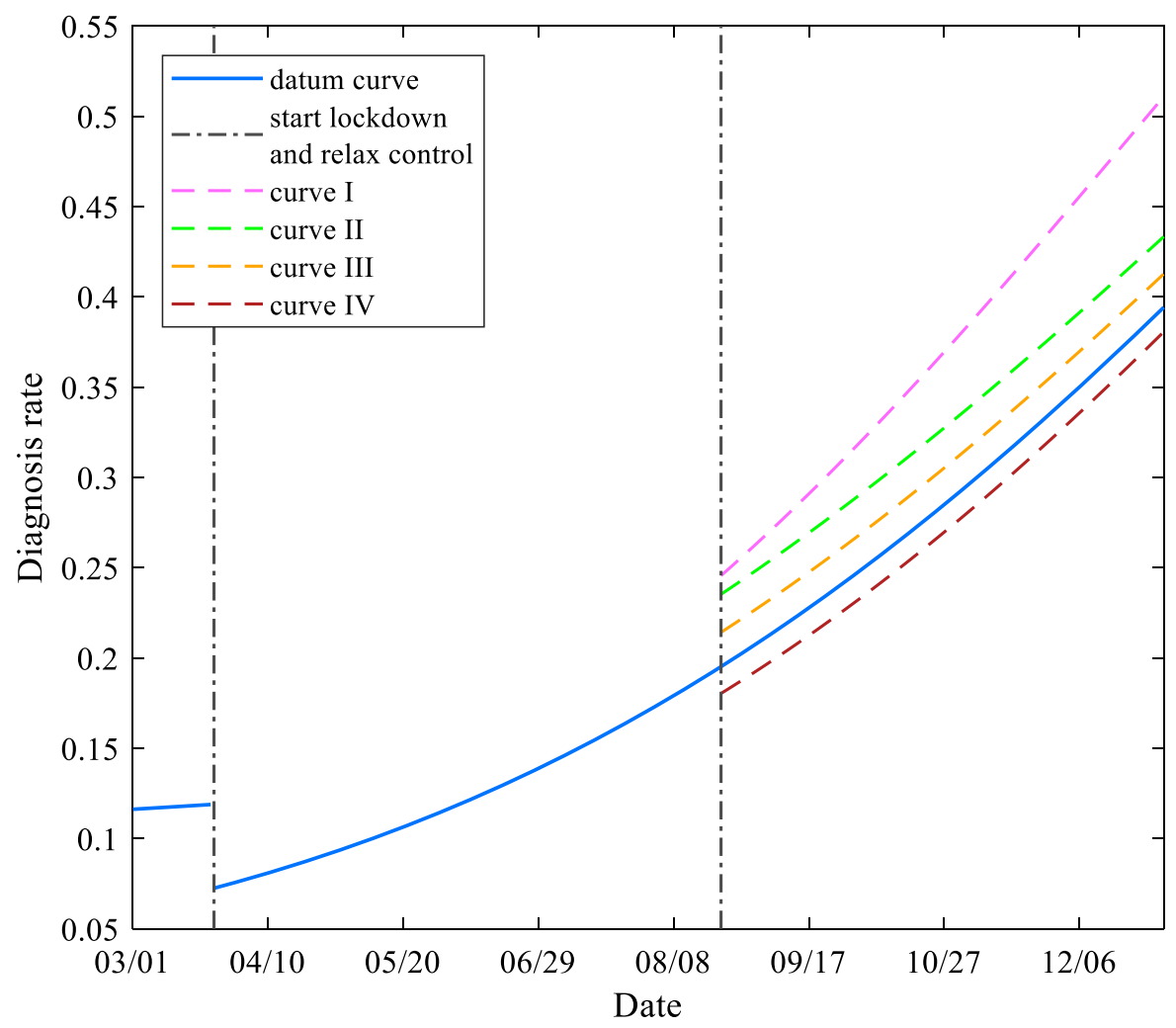

Fig. 8 Diagnosis rate curves under different parameters, for the combination of specific parameters $k_{\beta r}$ and $\tau_{\beta r}$, seeing Table 4

steeper as time goes. This phenomenon can be understood as Curves II, III and IV adjust the supply of the detection kit after unsealing, and Curve I is to accelerate the detection speed when the detection kit is sufficient.

Figure 9 depicts the impact of different diagnosis rates $\beta(t)$ on daily confirmed cases, cumulative confirmed cases and cumulative fatal cases after a further relaxation of control. The blue dotted line is the second peak curve after the $60 \%$ relaxation policy is adopted, which is used as the baseline for comparison. The diagnosis rate parameters of Curves I, II, III and IV are the parameters given in Fig. 8, respectively, and the remaining parameters are consistent with the baseline.

In Fig. 9a, after further relaxing the control, with the improvement of medical quarantine capacity, the peak time of the second peak of the newly confirmed case curve and the duration of the epidemic gradually shorten. Still, the peak of the second outbreak seems to decrease first and then increase. This phenomenon is because if the diagnosis rate is increased to a certain degree after unsealing, a large number of infectors in the population will be diagnosed and quarantined in a short time, and newly confirmed cases indeed rise rapidly in a short time. By comparing Curve I and Curve II, it is noted that Curve I can converge to zero significantly faster than Curve II. This is because the slope of the diagnosis rate Curve $\mathrm{I}$ is larger than that of Curve II over time [Fig. 8]. Therefore, fast testing speed plays a crucial role in promoting the elimination of the epidemic. The comparison between Curve IV and the baseline shows that if the medical detection ability cannot keep up with the blockade period after unblocking, it will take the risk that the second outbreak is even more severe than the first outbreak. Besides, in Fig. 9b and c, compared with the baseline, the final stable values of the cumulative diagnosed cases of Curves I, II and III were reduced by $29.731 \%$, $26.54 \%$ and $19.38 \%$, respectively, and the cumulative death cases reduced by $9.103 \%, 8.027 \%$ and $5.790 \%$, respectively. The diagnosis rate of Curve IV is only an average of $5.425 \%$ lower than the baseline, but the 
(a)

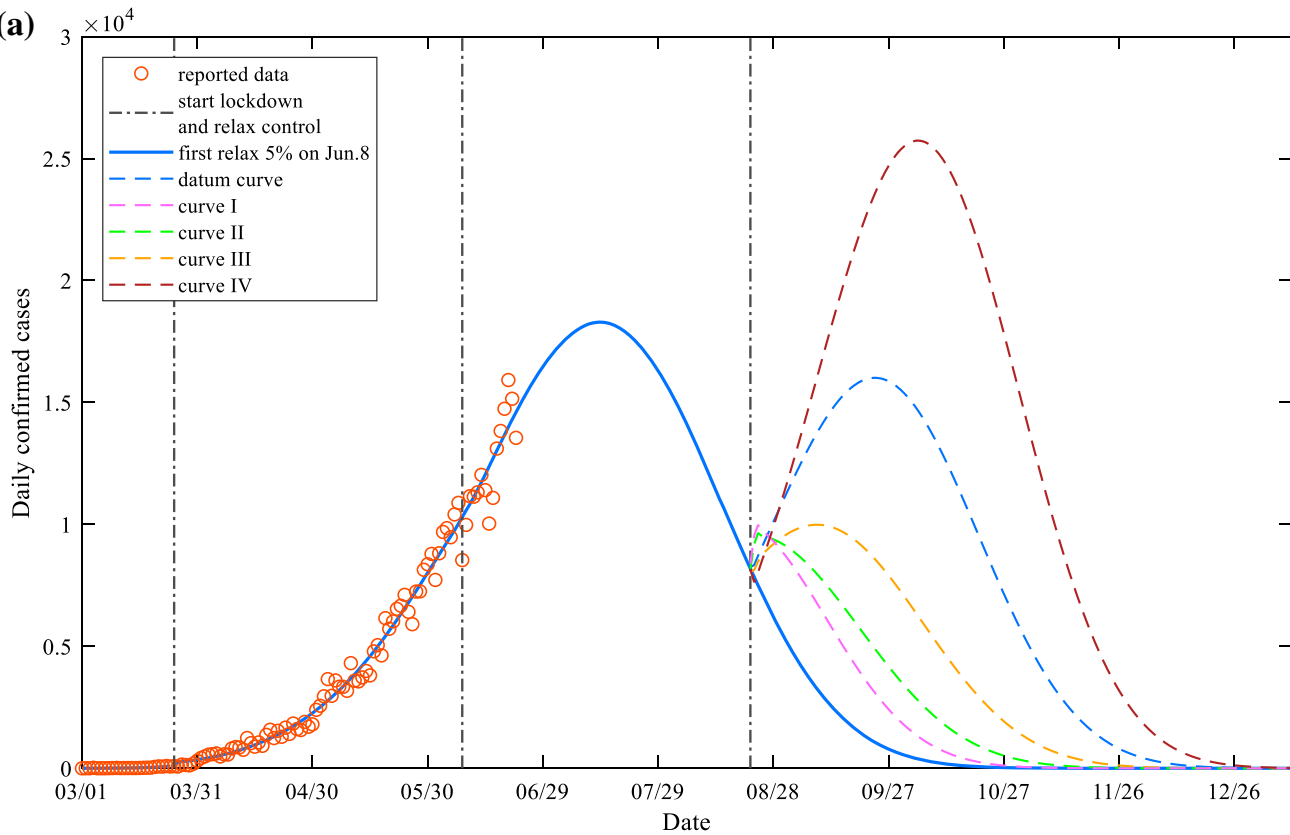

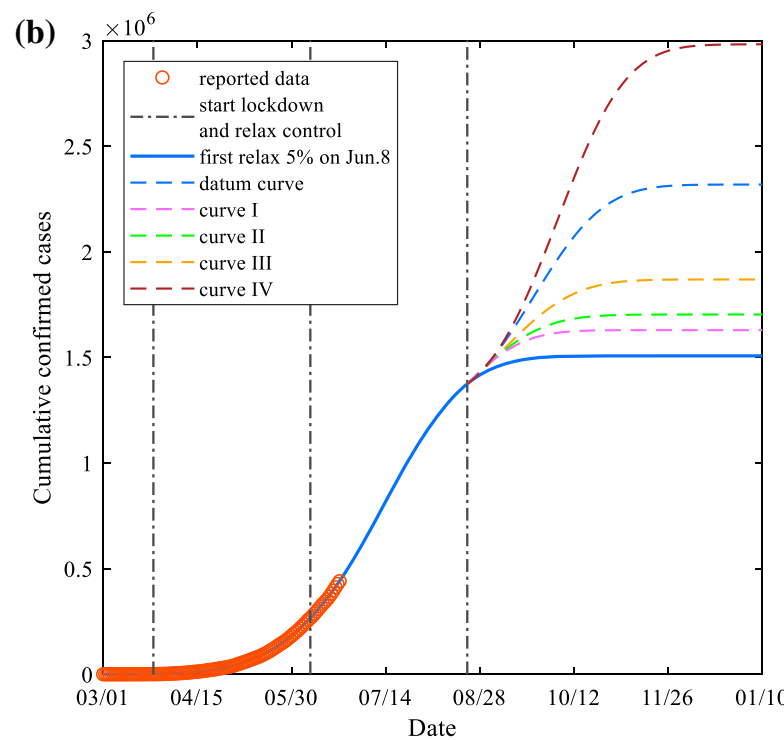

Fig. 9 Effect of different diagnosed rates on a daily confirmed cases, b cumulative confirmed cases and c cumulative fatal cases after a further relaxation of control. The higher the medical quarantine capability, the more capable of suppressing

final stable values of cumulative confirmed cases and cumulative fatal cases have increased by $28.64 \%$ and $7.357 \%$ on the basis of the baseline, which is close to the percentage reduction of Curve II. It can be seen that after the relaxation of control, the lack of medical quarantine capacity will cause more severe

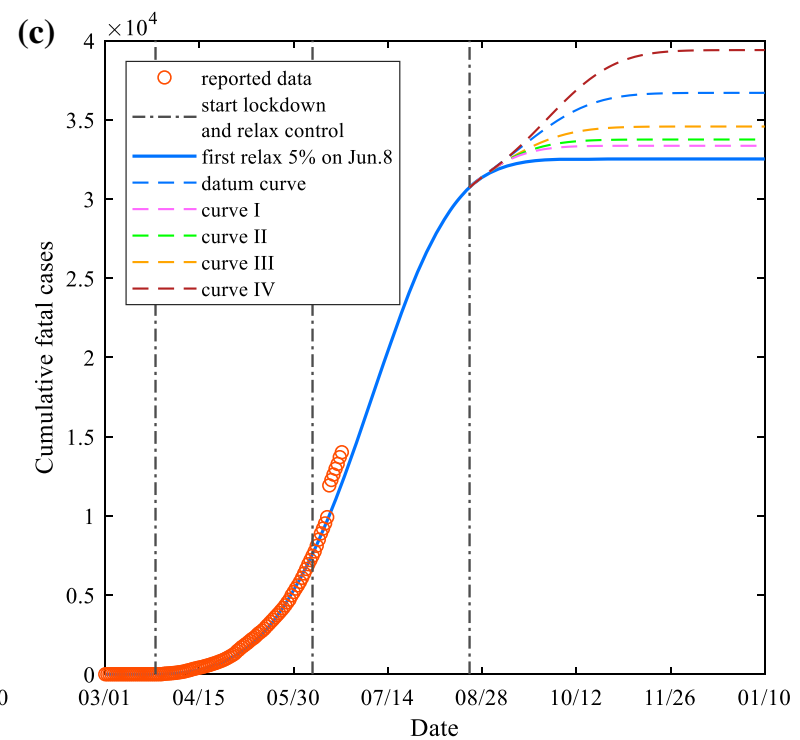

second outbreak. However, if the medical quarantine capacity is lower than the current one after the control is relaxed, it will cause more serious consequences

consequences. The specific data comparison is given in Table 5.

The results indicate that medical quarantine capacity is an important indicator after the relaxation of control, and it is also a criterion for evaluating whether to relax the control measures further. After 
Table 4 Details of the diagnosis rate curves in Fig. 8

\begin{tabular}{llll}
\hline Diagnosis rate curve number & $k_{\beta r}$ & $\tau_{\beta r}$ & $\begin{array}{l}\text { Average increase rate } \\
\text { compared to baseline }(\%)\end{array}$ \\
\hline I & 0.00890 & 276.1 & 28.97 \\
II & 0.00695 & 319.6 & 15.04 \\
III & 0.00723 & 329.8 & 7.158 \\
Baseline & 0.00754 & 338.00666 & - \\
IV & 0.00784 & 343.1 & -5.425 \\
\hline
\end{tabular}

Table 5 Impact of different diagnosis rates on the epidemic after a further relaxation of control

\begin{tabular}{|c|c|c|c|c|c|c|}
\hline \multirow{2}{*}{$\begin{array}{l}\text { Curve } \\
\text { number }\end{array}$} & \multirow{2}{*}{$\begin{array}{l}\beta(t) \text { average increase rate } \\
\text { compared to baseline }(\%)\end{array}$} & \multicolumn{3}{|c|}{ Daily confirmed cases curve } & \multirow{2}{*}{$\begin{array}{l}\text { Cumulative confirmed } \\
\text { cases curve } \\
\text { Stable value (decrease } \\
\text { rate compared to baseline) }\end{array}$} & \multirow{2}{*}{$\begin{array}{l}\text { Cumulative fatal cases } \\
\text { curve } \\
\text { Stable value (decrease } \\
\text { rate compared to baseline) }\end{array}$} \\
\hline & & $\begin{array}{l}\text { Second } \\
\text { peak } \\
\text { value }\end{array}$ & $\begin{array}{l}\text { Second } \\
\text { peak } \\
\text { time }\end{array}$ & $\begin{array}{l}\text { Second } \\
\text { outbreak } \\
\text { Duration }\end{array}$ & & \\
\hline I & 28.97 & 9976 & 2 & 95 & $1,629,180(29.73 \%)$ & $33,360(9.103 \%)$ \\
\hline II & 15.04 & 9649 & 2 & 114 & $1,703,095(26.54 \%)$ & $33,755(8.027 \%)$ \\
\hline III & 7.158 & 9985 & 17 & 129 & $1,869,139(19.38 \%)$ & $34,576(5.790 \%)$ \\
\hline Baseline & - & 16,007 & 32 & 144 & $2,318,550(-)$ & $36,701(-)$ \\
\hline IV & -5.425 & 25,733 & 44 & 156 & $2,982,492(-28.64 \%)$ & $39,401(-7.357 \%)$ \\
\hline
\end{tabular}

the implementation of the relaxation policy, if the supply of kits and the detection speed can be increased, then the second outbreak of epidemic can be avoided effectively, the duration of the epidemic can be shortened, and the number of deaths will be reduced relatively. However, if the medical quarantine capacity after deregulation is insufficient to reach the current level, it will cause a more serious secondary peak.

\subsection{Effect of public cooperation on the second outbreak}

The degree of public cooperation after deregulation can be understood as the extent to which the public complies with the government's epidemic prevention and control policies, including the social distance and mask order required by the government after the unblocking.

In fact, what is directly related to people's cooperation is the transmission rate $\alpha(t)$ in our model. In an ideal situation, if all people can strictly abide by government policies after loosening control, it will greatly reduce the spread of the virus. We assume that in this case, the people's maximum cooperation is $100 \%$, and the transmission rate will drop to the value $\alpha_{l}$ as in the lockdown period.

On the contrary, if all the people do not abide by the regulations after the unblocking, the people's minimum cooperation is $0 \%$, and the transmission rate will increase to the value $\alpha_{u}$ before the blockade. Therefore, after the relaxation of control, the current degree of people's cooperation $m \%$ is negatively correlated with the degree of relaxation $n \%$. Specifically, the more the government relaxes, the more the public will subjectively think that the epidemic is about to end, and its cooperation will be reduced accordingly. The public cooperation degree $m \%$ and relaxation degree $n \%$ can be expressed by the following relationship.

$m \%+n \%=1$

Therefore, the transmission rate parameter $\alpha_{r}$ after relaxing the control is also updated, as shown in Eq. (10).

$$
\alpha_{r}=\alpha_{l}+\left(\alpha_{u}-\alpha_{l}\right) \times n \%=\alpha_{l} \times m \%+\alpha_{u} \times n \%
$$

We selected India to adopt a policy of a further relaxing $45 \%$ of controls on August 22 as a 


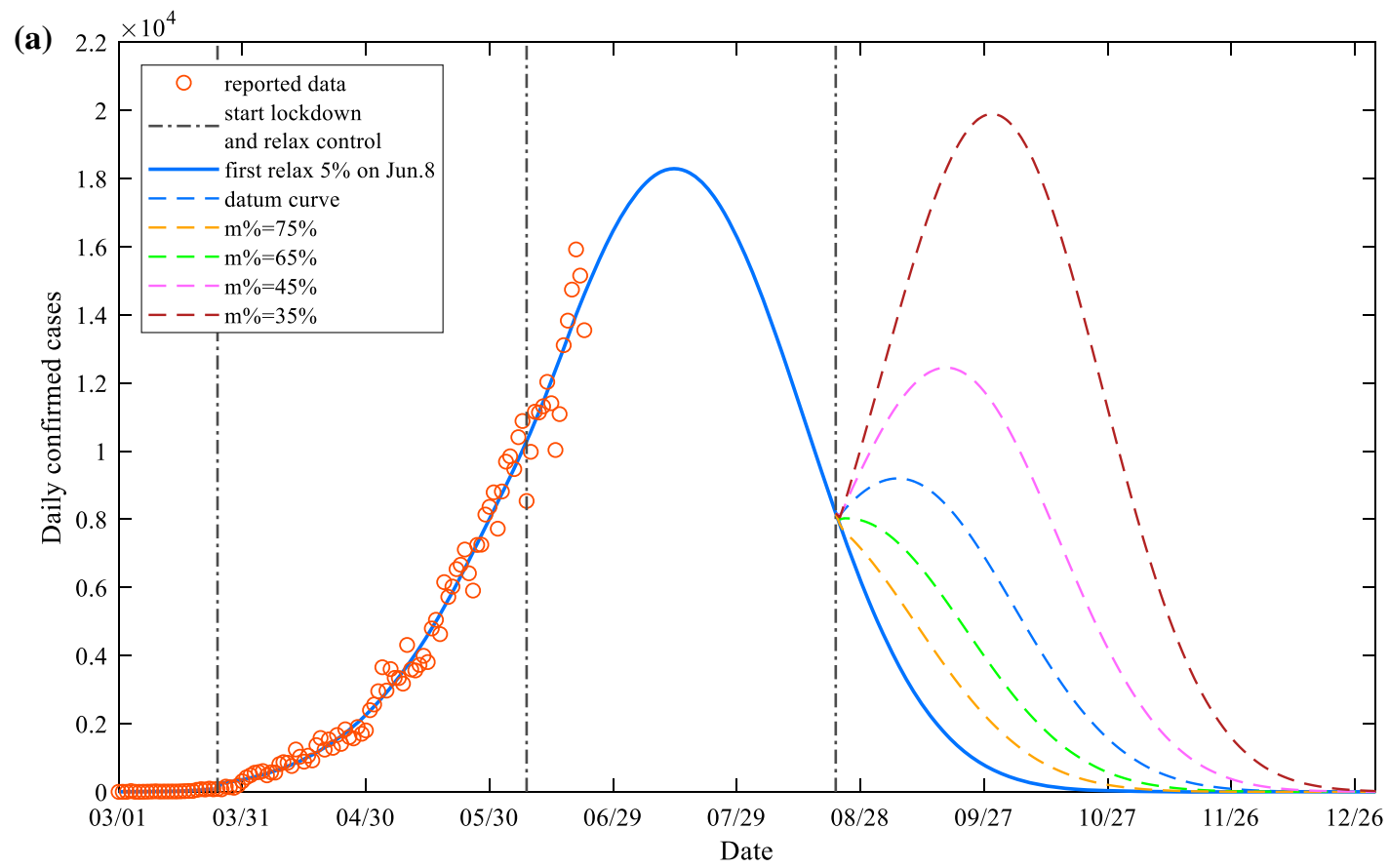

(b)

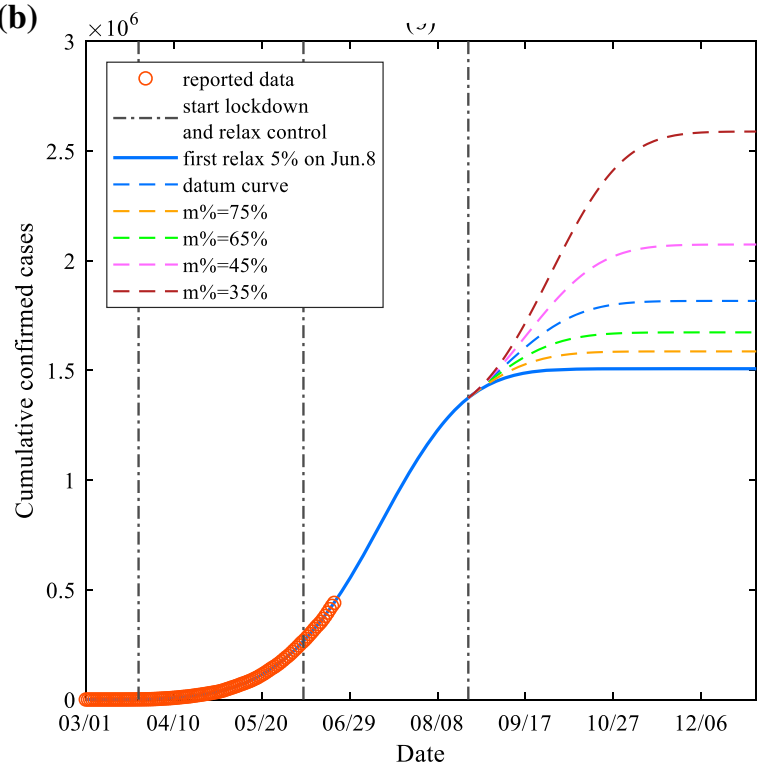

Fig. 10 Effect of different degrees of public cooperation on a daily confirmed cases, b cumulative confirmed cases and c cumulative fatal cases after a further relaxation of control.

benchmark to study the impact of public cooperation on the epidemic after relaxing control. Relaxing $45 \%$ control means that the current public cooperation is $55 \%$. Figure 10 shows the impact of different degrees of public cooperation on the daily confirmed cases, (c)

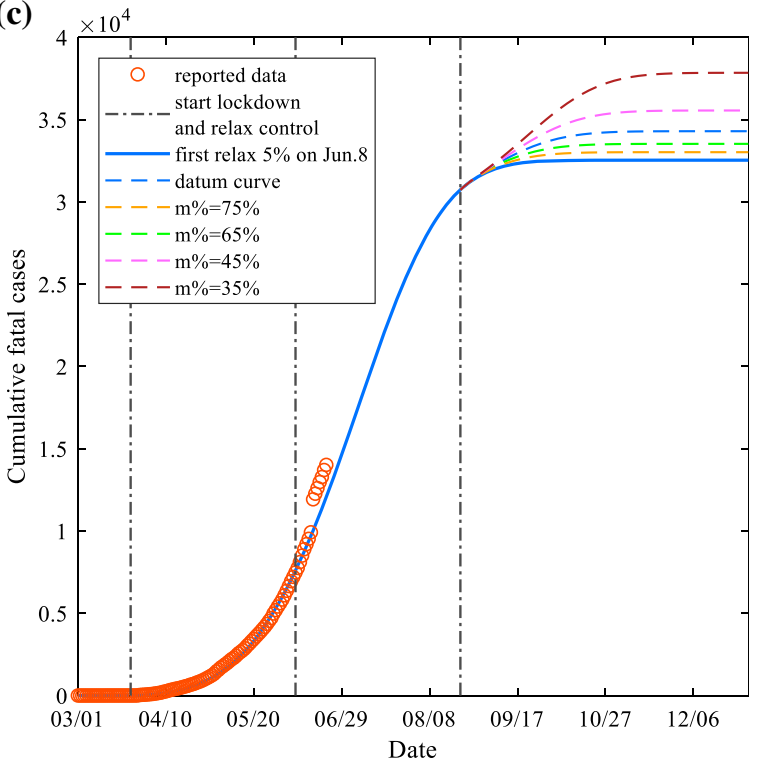

After the relaxation of control, the increase in public cooperation $m \%$ can also suppress the second outbreak of the pandemic and shorten the duration of the pandemic

cumulative confirmed cases and cumulative fatal cases. After a further relaxing control, as the people's cooperation increased from $35 \%$ to $75 \%$, the peak values of the secondary peak of new cases are gradually decreased, and the peak time gradually 
shortened until the secondary peak disappeared. The number of days with zero new cases and the duration of the epidemic are also gradually shortened; the final stable values of cumulative confirmed cases and cumulative fatal cases are also gradually reduced.

It can be seen that the degree of public cooperation is also a significant indicator after the relaxation of control, and its improvement does indeed have an excellent inhibitory effect on the second outbreak of the epidemic.

\section{Conclusion}

More and more countries have entered the rehabilitation phase of the COVID-19 pandemic and gradually implemented a loose control policy. According to the official daily report data, India is still in the rising stage and has not yet reached the inflection point. However, due to economic pressure, the Indian government had to adopt a relaxation policy in advance. In this study, we used the SIHRbased compartment model to study the development of COVID-19 in India after relaxing control. Sigmoid function with the cumulative effect was used in the model to characterize the diagnosis rate, cure rate and mortality rate. Meanwhile, considering the effect of lockdown on model parameters, the parameters in the model were designed as piecewise functions. We divided the reported data of India into pre-blocking and post-blocking and used the least squares method to fit. To compare with the Sigmoid function used, we also set the parameters as constant values for fitting. The goodness of fit indicates that the fitting results obtained using the Sigmoid function are generally better than those obtained with constant parameters.

The research results show that before the inflection point, even if the degree of relaxation is small, the cumulative number of confirmed cases and deaths will increase significantly. Therefore, it is suggested that no relaxation should be taken before the inflection point for any country's government. After reaching the inflection point, the degree of relaxation can be appropriately increased at a small price. But if the relaxation is too high or too early, there will be a second peak, which will lead to the second outbreak of the epidemic. To prevent the possible second outbreak, the impact of medical quarantine capacity and public cooperation on the second outbreak after the relaxation of control was studied, respectively. Both the medical quarantine capacity and the cooperation degree of the people play a vital role after the unblocking. Their improvements can effectively suppress the second outbreak and shorten the duration of the epidemic. Conversely, if the medical quarantine capacity and the degree of public cooperation after relaxation are lower than the level at the time of relaxing, it will lead to a more secondary severe outbreak, resulting in more deaths, putting massive pressure on the health care systems and economics.

The model and research we have proposed are not only applicable to India, but also provide a valuable direction for the formulation of policies in other countries, and are of constructive significance.

Acknowledgements This work was supported by the National Natural Science Foundation of China (61873186).

\section{Compliance with ethical standards}

Conflict of interest The authors declare that they have no conflict of interest.

\section{References}

1. Momtazmanesh, S., Ochs, H.D., Uddin, L.Q., Perc, M., Routes, J.M., et al.: All together to fight COVID-19. Am. J. Trop. Med. Hyg. 102(6), 1181-1183 (2020)

2. World Health Organization: Coronavirus disease (COVID2019) Situation reports (2020). https://www.who.int/emer gencies/diseases/novel-coronavirus-2019/situation-reports/ . Accessed 22 June 2020

3. Chan, J.F., Yuan, S., Kok, K., To, K.K., Chu, H., Yang, J., et al.: A familial cluster of pneumonia associated with the 2019 novel coronavirus indicating person-to-person transmission: a study of a family cluster. Lancet 395, 514-523 (2020)

4. Tong, Z., Tang, A., Li, K., Li, P., Wang, H., Yi, J., et al.: Potential presymptomatic transmission of SARS-CoV-2, Zhejiang Province, China, 2020. Emerg. Infect. Dis. 26, 1052-1054 (2020)

5. Centers for disease control and prevention: 2019 novel coronavirus (2020). https://www.cdc.gov/coronavirus/ 2019-ncov/symptoms-testing/symptoms.html. Accessed 22 Jun 2020

6. Wilder-Smith, A., Freedman, D.: Isolation, quarantine, social distancing and community containment: pivotal role for old-style public health measures in the novel coronavirus (2019-nCoV) outbreak. J. Travel Med. (2020). https://doi.org/10.1093/jtm/taaa020

7. India Covid-19 tracker (2020). https://www.covid19india. org/. Accessed 22 June 2020 
8. India Government Inter-Ministerial Notifications (2020). https://covid19.india.gov.in/documents/. Accessed 22 June 2020

9. Helbing, D., Brockmann, D., Chadefaux, T., Donnay, K., Blanke, U., et al.: Saving human lives: what complexity science and information systems can contribute. J. Stat. Phys. 158(3), 735-781 (2015)

10. Perc, M., Miksić, N.G., Slavinec, M., Stožer, A.: Forecasting COVID-19. Front. Phys. 8, 127 (2020)

11. Fang, Y., Nie, Y., Penny, M.: Transmission dynamics of the COVID-19 outbreak and effectiveness of government interventions: a data-driven analysis. J. Med. Virol. 92, 645-659 (2020)

12. Wu, J.T., Leung, K., Leung, G.M.: Nowcasting and forecasting the potential domestic and international spread of the 2019-nCoV outbreak originating in Wuhan, China: a modelling study. Lancet 395, 689-697 (2020)

13. Anastassopoulou, C., Russo, L., Tsakris, A., Siettos, C.: Data-based analysis, modeling and forecasting of the COVID-19 outbreak. PLoS ONE 15(3), e0230405 (2020)

14. Zhao, S., Chen, H.: Modeling the epidemic dynamics and control of COVID-19 outbreak in China. Quant. Biol. 8(1), 11-19 (2020)

15. Rong, X., Yang, L., Chu, H., Fan, M.: Effect of delay in diagnosis on transmission of COVID-19. Math. Biosci. Eng. 17(3), 2725-2740 (2020)
16. Mandal, M., Jana, S., Nandi, S.K., Khatua, A., Adak, S., Kar, T.K.: A model based study on the dynamics of COVID-19: prediction and control. Chaos Soliton Fract. 136, 109889 (2020)

17. Huang, J., Qi, G.: Effects of control measures on the dynamics of COVID-19and double-peak behavior in Spain. Nonlinear Dyn. (2020). https://doi.org/10.1007/s11071020-05901-2

18. Kermack, W.O., McKendrick, A.G.: A contribution to the mathematical theory of epidemics. Proc. R. Soc. Lond. A 115, 700-721 (1927)

19. Lauer, S.A., Grantz, K.H., Bi, Q., Jones, F.K., Zheng, Q., et al: The incubation period of coronavirus disease 2019 (COVID-19) from publicly reported confirmed cases: estimation and application. Ann. Intern. Med. 172(9), 577582 (2020)

20. World Population Dashboard (2020). https://www.unfpa. org/data/world-population-dashboard. Accessed 22 June 2020

Publisher's Note Springer Nature remains neutral with regard to jurisdictional claims in published maps and institutional affiliations. 